\title{
Assistência de enfermagem na prevenção da lesão por pressão: uma revisão integrativa
}

\author{
Nursing care in the prevention of pressure: an integrative review \\ Asistencia de enfermería en la prevención de la lesión por presión: una revisión \\ integrativa
}

Francinalva de Almeida², Maria dos Milagres Santos da Costa ${ }^{2}$, Ellen Eduarda Santos Ribeiro ${ }^{1 *}$, Danielle Christina de Oliveira Santos ${ }^{2}$, Nara Daniele Alcântara Silva², Rosilda Evangelista da Silva², Kamilla Pinheiro Saraiva², Polyana Coutinho Bento Pereira ${ }^{3}$.

\section{RESUMO}

Objetivo: analisar a produção científica sobre a assistência de enfermagem com vista à prevenção de lesão por pressão em pacientes hospitalizados. Método: Foi realizada uma revisão integrativa de literatura a partir de banco de dados: LILACS, MEDLINE e SCIELO, através da procura de produção científica do período de 2010 a 2017. Foram encontradas 200 publicações indexadas no banco de dados das citadas fontes. Desses artigos foram separados os que realmente atendiam aos critérios de inclusão: artigos na íntegra que tratem da assistência de enfermagem na prevenção e tratamento de lesão por pressão em pacientes hospitalizados, nos idiomas português e inglês. Já os critérios de exclusão foram artigos que abordassem outros assuntos da lesão por pressão, assim como artigos que fossem anteriores a 2010. Como refino da seleção, apenas 11 foram incluídos na pesquisa. Resultados: evidenciaram a importância de padronizar as notificações e qualificar a equipe de enfermagem para atuar de forma mais eficaz na intervenção da temática abordada. Conclusão: Os dados mostraram que a prevenção e o tratamento das lesões por pressão estão diretamente ligados aos cuidados da enfermagem.

Descritores: Assistência de Enfermagem, Lesão por Pressão, Hospitais.

\begin{abstract}
Objective: To analyze the scientific production on nursing care with a view to preventing pressure injury in hospitalized patients. Method: An integrative revision of literature was conducted from the database: LILACS, MEDLINE and SCIELO, through the search for scientific production of the period from 2010 to 2017. 200 indexed publications were found in the database of the aforementioned sources. These articles were separated from those who actually attended the inclusion criteria: articles in the integra that treat nursing assistance in preventing and treating pressure lesions in hospitalized patients, in languages, Portuguese and English. The exclusion criteria have been articles covering other subjects of the pressure lesion, as well as articles that are prior to 2010. As refining the selection only 11 were included in the survey. Results: Evidenced the importance of standardising the notifications and qualifying the nursing staff to act more effectively in the intervention of the thematic discussed. Conclusion: The data showed that the prevention and treatment of pressure lesions is directly linked to nursing care.
\end{abstract}

Descriptors: Nursing assistance, Pressure lesion, Hospitals.

\footnotetext{
${ }^{1}$ Discente do Departamento de Enfermagem pela Universidade Federal do Piauí (UFPI), Teresina, Piauí. ${ }^{\star}$ E-mail: ellenribeirosr@gmail.com

${ }^{2}$ Graduação em Enfermagem pela Associação de Ensino Superior do Piauí (AESPI), Teresina, Piauí.

${ }^{3}$ Graduação em Enfermagem pela Universidade Estadual do Piauí (UESPI), Teresina, Piauí
}

SUBMETIDO EM: 1/2019

ACEITO EM: 2/2019

PUBLICADO EM: $8 / 2019$

REAS/EJCH | Vol.Sup.30 | e1440 | DOI: https://doi.org/10.25248/reas.e1440.2019 Página 1 de 9 


\section{RESUMEN}

Objetivo: analizar la producción científica sobre cuidados de enfermería con el fin de prevenir lesiones por presión en pacientes hospitalizados. Método: Se realizó una revisión integrativa de literatura a partir de base de datos: LILACS, MEDLINE y SCIELO, a través de la demanda de producción científica del período de 2010 a 2017. Se encontraron 200 publicaciones indexadas en la base de datos de las citadas fuentes. Estos artículos fueron separados los que realmente cumplieron con los criterios de inclusión: artículos completos tratar con los cuidados de enfermería en la prevención y tratamiento de úlceras por presión en pacientes hospitalizados, en portugués y en inglés. Los criterios de exclusión fueron artículos que abordaran otros asuntos de la lesión por presión, así como artículos que fueran anteriores a 2010. Como refinación de la selección, sólo 11 fueron incluidos en la investigación. Resultados: evidenciaron la importancia de estandarizar las notificaciones y calificar al equipo de enfermería para actuar de forma más eficaz en la intervención de la temática abordada. Conclusión: Los datos mostraron que la prevención y el tratamiento de las lesiones por presión están directamente ligados a los cuidados de la enfermería.

Palabras clave: Asistencia de Enfermería, Lesión por Presión, Los hospitales.

\section{INTRODUÇÃO}

Segundo Silva RCL (2011); a ocorrência de lesões por pressão (LPP) em pacientes internados é considerada não só no Brasil, mas também mundialmente como problema grave, especialmente em pessoas idosas e em pacientes portadores de doenças crônico-degenerativas.

O paciente hospitalizado por ter sua condição fisiológica comprometida pela patologia apresentada, necessita de cuidados de aspectos físicos, psicológicos e sociais, ou seja, um cuidar de forma integral. Dessa forma, é imprescindível a qualidade da assistência a ser prestada a esse paciente (SILVA RCL, 2011).

A definição de Lesões por Pressão (LPP) são áreas de destruição tecidual produzidas pela compressão da pele contra as proeminências ósseas, principalmente os ossos do sacro, do trocânter e do ísquio, por conta que a superfície de contato tem duração por um tempo prolongado. Devido a isso, prejudica o fornecimento de sangue ao tecido e de nutrientes, levando à insuficiência vascular, anóxia tecidual e morte das células (DOMANSKY RC e BORGES EL, 2014).

As LPPs se desenvolvem em até em 24 horas ou podem levar até cinco dias para ocorrer. Diante disso, a equipe multidisciplinar de saúde devem ser responsáveis pela prevenção da lesão, com isso, devem conhecer os fatores de risco para formação da LPP, que são: perfusão tecidual, idade, imobilidade, atividade, nível de consciência, alguns medicamentos, umidade excessiva, nutrição, hidratação e algumas doenças crônicas como diabetes e cardiovasculares para que haja a redução da incidência das LPPs (DOMANSKY RC e BORGES EL, 2014).

De acordo com Galvão NS, et al. (2017) a pressão mantida por um período de duas horas pode propiciar uma lesão isquêmica. Além disso, os pacientes com grave comprometimento do estado geral podem desenvolver lesões em tempo inferior a duas horas. Quando a pressão cutânea é superior à pressão capilar média $(32 \mathrm{mmHg})$ em indivíduos sadios, ocorre isquemia local, provocando edema, eritema, erosão e úlceras.

A LPP, como destaca Silva RCL (2011), se tornou um problema hospitalar que é bastante comum, isso ocorre principalmente quando idosos ficam por muito tempo hospitalizados e não possuem uma assistência adequada, ou seja, ficando expostos a complicações.

Além disso, os estudos demonstram que as LPPs também ocorrem em casas de repouso ou domicílios devido ao desconhecimento da prevenção dessas lesões ou pelo desconhecimento de como tratar essa complicação. Diante disso, o tratamento é mais demorado, comprometendo a sua qualidade de vida do idoso.

Diante do que foi apresentado, esse problema tenderá a crescer visto que há um aumento significativo na população idosa (FERNANDES LM e CALIRI HML, 2016). 
Portanto, é necessário que a equipe de enfermagem esteja preparada e disposta a dar um cuidado intensivo a estes pacientes com o objetivo de aumentar a qualidade da assistência, além, de promover ações preventivas com intuito de diminuir os indicadores de LPPs.

Sendo assim, o presente estudo tem como objetivo avaliar o conhecimento e a produtividade cientifica acerca do papel do enfermeiro diante dos cuidados de enfermagem na prevenção de lesão por pressão, além de reunir evidencias científicas sobre essa assistência.

\section{METODOLOGIA}

O estudo está caracterizado como uma revisão integrativa da literatura. Este método possibilita sumarizar as pesquisas publicadas e obter conclusões a partir da pergunta norteadora (MENDES KDS, et al., 2008). A revisão integrativa da literatura é desenvolvida a partir de material já elaborado e publicado, constituído principalmente de revistas e artigos científicos. Sua finalidade é colocar o pesquisador em contato com o que foi produzido a respeito do seu tema de pesquisa (MARCONI MA e LAKATOS EM, 2010). A questão norteadora que possibilitou realizar o estudo foi o que a literatura evidencia sobre a assistência de enfermagem com vista à prevenção de lesão por pressão?

O levantamento bibliográfico para a pesquisa foi realizado por meio de indexadores online, que se encontram referenciados na Literatura Científica e Técnica da América Latina e Caribe (LILACS), Sistema Online de Busca e Análise de Literatura Médica (MEDLINE), Scientific Eletronic Library Online (SCIELO).

Os dados foram coletados no período de setembro de 2017, através de levantamento bibliográfico realizado em trabalhos publicados nas bases de dados: LILACS, MEDLINE e SCIELO, cujo período de publicação será de 2010 a 2017, utilizando-se os descritores: Assistência de Enfermagem, Lesão por Pressão, Hospitais, todos cadastrados nos Descritores em Ciência da Saúde (DECS).

Foram incluídos na pesquisa artigos na íntegra que tratem da assistência de enfermagem à pacientes hospitalizados com lesão por pressão e nos idiomas português e inglês. Já os critérios de exclusão serão artigos que abordem outros tipos de lesões, assim como artigos que sejam anteriores a 2010.

Ademais, foram realizadas duas etapas seguindo a orientação de Minayo MCS (2013), a primeira fase a pré-análise que consiste na escolha dos artigos que foram analisados; retomada das hipóteses e dos objetivos inclusos inicialmente, e posteriormente a reformulação frente ao material coletado através de um formulário (Apêndice A) contendo identificação do artigo para que possam elaborar os indicadores que orientem a interpretação final.

No processamento e análise dos dados para a seleção dos artigos foram observadas algumas características dos estudos, através de um formulário contendo identificação do artigo (autores, delineamentos, objetivos, população estudada e resultados).

A síntese dos dados extraídos dos artigos foi apresentada de forma descritiva em tabelas e quadros, reunindo o conhecimento produzido sobre o assunto investigado na presente revisão integrativa.

\section{RESULTADOS}

Para a coleta de dados, foi lido cada título e resumido exaustivamente para ter a confirmação se estes contemplavam a pergunta norteadora desta investigação e se atendiam aos critérios de exclusão e inclusão. A busca dessa estratégia foi evidenciada no Quadro 1.

Após o levantamento dessas publicações cientificas, mencionando os descritores selecionados partiu-se para a seleção dos artigos.

Das 200 referências encontradas na base de dados BVS (biblioteca virtual em saúde), 90 foram de idiomas não complementados nos critérios de inclusão e $53 \mathrm{com}$ duplicidades. Das 57 publicações restantes, 45 artigos não eram de publicações anteriores a 2010. 
Quadro 1 - Publicações encontradas nas bases de dados, LILACS, MEDLINE e SCIELO, com as palavras chaves estabelecidas.

\begin{tabular}{|l|c|c|c|}
\hline DESCRITORES & LILACS & MEDLINE & SCIELO \\
\hline Assistência de Enfermagem & 10 & 18 & 25 \\
\hline Lesão por Pressão & 12 & 18 & 25 \\
\hline Hospitais & 19 & 23 & 46 \\
\hline
\end{tabular}

Fonte: Pesquisa direta, 2017.

Os resultados apresentados a seguir, são oriundos da amostragem dos 12 (doze) artigos que atenderam aos critérios de inclusão e aos objetivos de estudo. Para uma melhor compreensão, foram distribuídos conforme a Tabela 1.

Tabela 1- Distribuição dos artigos que tratam sobre a assistência de enfermagem com vista à prevenção de lesão por pressão, segundo o ano de publicação, banco de dados, periódicos de publicação, abordagens e titulação dos autores. Teresina-PI, 2017.

\begin{tabular}{|c|c|c|}
\hline Especificação da variável & N.- Absoluto & Percentual (\%) \\
\hline \multicolumn{3}{|l|}{ Ano de publicação } \\
\hline 2010 & 1 & 9,1 \\
\hline 2011 & 0 & 0,0 \\
\hline 2012 & 2 & 18,1 \\
\hline 2013 & 1 & 9,1 \\
\hline 2014 & 1 & 9,1 \\
\hline 2015 & 5 & 45,5 \\
\hline 2016 & 1 & 9,1 \\
\hline \multicolumn{3}{|l|}{2017} \\
\hline Subtotal & 11 & 100 \\
\hline \multicolumn{3}{|l|}{ Bancos de Dados } \\
\hline LILACS & 2 & 18,1 \\
\hline MEDLINE & 4 & 36,4 \\
\hline SCIELO & 5 & 45,5 \\
\hline Subto Gtal & 11 & 100 \\
\hline \multicolumn{3}{|l|}{ Periódicos } \\
\hline Acta paul. Enferm. & 1 & 9,1 \\
\hline Esc. Anna Nery & 1 & 9,1 \\
\hline Invest Educ Enferm. & 1 & 9,1 \\
\hline Journal Health NPEPS & 2 & 18,1 \\
\hline Rev. Latino-Am. Enfermagem & 1 & 9,1 \\
\hline Revista Lume digital & 1 & 9,1 \\
\hline Rev Bras Enferm & 4 & 36,4 \\
\hline \multicolumn{2}{|l|}{ Abordagem } & 100 \\
\hline Qualitativa & 9 & 81,9 \\
\hline Quantitativa & 2 & 18,1 \\
\hline \multicolumn{3}{|l|}{ Titulação dos Autores } \\
\hline Graduado & 1 & 9,1 \\
\hline Especialista & 2 & 18,1 \\
\hline Mestre & 1 & 9,1 \\
\hline Doutor & 2 & 18,1 \\
\hline Pós-Doutor & 1 & 9,1 \\
\hline \multirow[t]{2}{*}{ Não-Especificado } & 1 & 9,1 \\
\hline & 3 & 27,4 \\
\hline Subtotal & 11 & 100 \\
\hline
\end{tabular}

Fonte: Pesquisa direta, 2017. 
Os Quadros 1 e 2 apresentados anteriormente evidenciam uma amostra diversificada entre a quantidade e os sujeitos da pesquisa, constituindo uma amostra significativa entre enfermeiros. Observou-se que, em relação às intervenções para coleta de dados, os instrumentos utilizados foram diversificados, apresentando tanto a entrevista semiestruturada, quanto o uso de questionários e/ou formulários e revisão integrativa da literatura.

Das publicações que restaram apenas 11 possuem a temática sobre a assistência de enfermagem com vista à prevenção de lesão por pressão, tendo como guia os objetivos da pesquisa associados aos critérios de inclusão. Foi realizada a leitura minuciosa de todos os capítulos e separados aqueles que tinham relação os objetivos da pesquisa.

No que tange aos desfechos finais, a análise é positiva, porque foi possível analisar a produção científica sobre a assistência da enfermagem na prevenção de lesão por pressão em hospitais. Entretanto, as amostras evidenciaram a importância de padronizar as notificações e qualificar a equipe de enfermagem para atuar de forma mais eficaz na intervenção da temática abordada.

Após leitura minuciosa dos artigos e compreensão de seus objetos de estudo, foi possível classificá-los em duas categorias (Quadro 3), a partir da similaridade semântica entre as pesquisas, sendo estas: A incidência da laserterapia no tratamento de lesão por pressão em Unidade de Terapia Intensiva (UTI) e A importância da laserterapia no tratamento de lesão por pressão.

Com a pesquisa foram obtidos doze trabalhos científicos, após serem lidos na íntegra e distribuídos em um quadro de forma resumida as seguintes áreas: atuação, autores, ano, títulos, resultados e conclusão. Os artigos foram enumerados do número 01 ao 11 para facilitar a análise de identificação das etapas lidos agrupados em eixos temáticos.

Os resultados da análise da amostra do conteúdo dos 11 (onze) artigos estão apresentados no quadro 3 a seguir, em forma de duas categorias semânticas e segundo os autores dos artigos analisados.

Quadro 3 - Classificação dos artigos de acordo com as categorias, unidade de análise autores e ano.

\begin{tabular}{|c|c|c|c|}
\hline \multirow[t]{2}{*}{ CATEGORIAS } & \multicolumn{2}{|c|}{ UNIDADES DE ANÁLISE } & \multirow[t]{2}{*}{ AUTOR (ES), ANO } \\
\hline & $\mathrm{N}$ & $(\%)$ & \\
\hline $\begin{array}{l}\text { A assistência e cuidado da } \\
\text { enfermagem na prevenção de lesão } \\
\text { por pressão. }\end{array}$ & 6 & 54,6 & $\begin{array}{l}\text { LAMÃO LCL, et al. } \\
\text { (2016); FRANÇA JRG, et } \\
\text { al. (2016); } \\
\text { SAATKAMP F (2012), } \\
\text { SOUZA VPS, et al. (2016); } \\
\text { SANTOS CT et al. } \\
\text { (2013); LIMA AFC, et } \\
\text { al. (2016). }\end{array}$ \\
\hline $\begin{array}{l}\text { Incidência e prevalência de lesão } \\
\text { por pressão em pacientes } \\
\text { hospitalizados }\end{array}$ & 5 & 45,4 & $\begin{array}{l}\text { MATOS LS, et al. (2010); } \\
\text { OLKOSK E e ASSIS GM, } \\
\text { (2016); GALVÃO NS, et } \\
\text { al. (2017); SANTANA WS, } \\
\text { et al. (2014); ROGENSK } \\
\text { NMB e } \\
\text { KURCGANT P (2012). }\end{array}$ \\
\hline TOTAL & 11 & 100 & 11 \\
\hline
\end{tabular}

Fonte: Pesquisa direta, 2017. 
Quadro 2 - Distribuição das publicações que abordam a assistência de enfermagem com vista à prevenção de lesão por pressão de acordo com, autor/ano, objetivo, metodologia e resultados.

\begin{tabular}{|c|c|c|c|c|}
\hline № & Autor/ano & Título & Resultados & Conclusão \\
\hline 1 & $\begin{array}{l}\text { MATOS LS, et al. } \\
2010 .\end{array}$ & $\begin{array}{l}\text { Incidência e prevalência de } \\
\text { Úlcera por pressão no CTI de } \\
\text { um Hospital Público do DF. }\end{array}$ & $\begin{array}{l}\text { Os resultados evidenciaram que metade das } \\
\text { lesões apareceram entre o } 2^{\circ} \text { e o } 4^{\circ} \text { dia de } \\
\text { avaliação. Segundo a Escala de Braden, } \\
55,5 \% \text { dos pacientes apresentavam alto } \\
\text { risco de desenvolver LPP, destes, } 40 \% \\
\text { desenvolveram. }\end{array}$ & $\begin{array}{l}\text { Conclui-se que a assistência prestada aos } \\
\text { pacientes é desenvolvida através de } \\
\text { estratégias de redução destes agravos. }\end{array}$ \\
\hline 2 & LAMÃO LCL, et al. 2016 & $\begin{array}{l}\text { Cuidados de enfermagem na } \\
\text { prevenção de Lesão por } \\
\text { Pressão. }\end{array}$ & $\begin{array}{l}\text { Os resultados evidenciaram que a } \\
\text { enfermagem se faz necessária juntamente } \\
\text { com a equipe multidisciplinar e uma } \\
\text { comissão de preventiva de lesão por } \\
\text { pressão para a adoção de protocolos de } \\
\text { avaliação de riscos e tratamento. }\end{array}$ & $\begin{array}{l}\text { Conclui-se que prevenção das lesões por } \\
\text { pressão está diretamente ligada aos } \\
\text { cuidados da enfermagem, dispondo de } \\
\text { medidas simples, porém eficazes. }\end{array}$ \\
\hline 3 & $\begin{array}{l}\text { FRANÇA JRG, et al. } \\
2016 .\end{array}$ & $\begin{array}{l}\text { Cuidados de Enfermagem na } \\
\text { Prevenção de Lesões por } \\
\text { Pressão em Unidades de } \\
\text { Terapia Intensiva. }\end{array}$ & $\begin{array}{l}\text { Os resultados evidenciaram que que o } \\
\text { gênero masculino apresentou uma } \\
\text { incidência maior para o risco de } \\
\text { desenvolvimento de lesões por pressão, } \\
\text { embora não existam dados na literatura que } \\
\text { justifiquem essa estatística. }\end{array}$ & $\begin{array}{l}\text { Conclui-se que a identificação dos fatores } \\
\text { determinantes da LPP é de extrema } \\
\text { importância para a prática, pois proporciona } \\
\text { um conhecimento de grande } \\
\text { relevância em âmbito hospitalar, visto que } \\
\text { as LPP constituem um potencial problema, } \\
\text { devido à longa permanência dos pacientes } \\
\text { e pelo grau de complexidade que os } \\
\text { mesmos apresentam. }\end{array}$ \\
\hline 4 & SAATKAMP F, 2012 & $\begin{array}{llr}\text { Cuidados de } & \text { enfermagem na } \\
\text { prevenção } & \text { a úlceras por } \\
\text { pressão } & \text { em } & \text { pacientes } \\
\text { hospitalizados. }\end{array}$ & $\begin{array}{l}\text { Os resultados demonstraram que a } \\
\text { enfermagem destaca como fatores para a } \\
\text { incidência de lesão por pressão a má } \\
\text { nutrição dos pacientes, assim como a falta } \\
\text { de conhecimento dos familiares sobre as } \\
\text { LPP. }\end{array}$ & $\begin{array}{l}\text { Conclui-se que a enfermagem cumpre seu } \\
\text { papel no cuidado e assistência para com o } \\
\text { paciente, entretanto esse cuidado dever ser } \\
\text { buscado por toda instituição hospitalar. }\end{array}$ \\
\hline 5 & $\begin{array}{l}\text { OLKOSK E e ASSIS } \\
\text { GM, } 2016\end{array}$ & $\begin{array}{l}\text { Aplicação de medidas de } \\
\text { prevenção para úlceras por } \\
\text { Pressão pela equipe de } \\
\text { enfermagem antes e após uma } \\
\text { campanha educativa. }\end{array}$ & $\begin{array}{l}\text { Os resultados demonstraram que a } \\
\text { aplicação de medidas simples como o } \\
\text { reposicionamento, angulação de } \\
\text { lateralização e elevação de cabeceira e } \\
\text { elevação de calcâneos, contribuem } \\
\text { para a redução de casos de LPP. }\end{array}$ & $\begin{array}{l}\text { Conclui-se que a efetividade deste tipo de } \\
\text { abordagem é dependente da } \\
\text { participação ativa da equipe na discussão } \\
\text { de medidas a serem aplicadas e de } \\
\text { recursos disponíveis. Faz-se necessária } \\
\text { uma abordagem contínua e sistemática. }\end{array}$ \\
\hline
\end{tabular}

REAS/EJCH | Vol.Sup.30 | e1440 | DOI: https://doi.org/10.25248/reas.e1440.2019 Página 6 de 9 


\begin{tabular}{|c|c|c|c|c|}
\hline 6 & SOUZA VPS, et al. 2016 & $\begin{array}{l}\text { Assistência de enfermagem na } \\
\text { prevenção de lesão por } \\
\text { pressão. }\end{array}$ & $\begin{array}{l}\text { Os resultados demonstraram que } \\
\text { Assistência de Enfermagem possui ações } \\
\text { determinantes na prevenção, promoção e } \\
\text { tratamento das LPP, sendo necessária a } \\
\text { atuação direta do enfermeiro para a } \\
\text { efetividade do tratamento preventivo, } \\
\text { moldando os cuidados conforme os fatores } \\
\text { Integrais e individuais. }\end{array}$ & $\begin{array}{l}\text { Conclui-se que é essencial que a equipe } \\
\text { multidisciplinar reforce as intervenções, } \\
\text { interagindo no planejamento e na } \\
\text { elaboração de programa de prevenção e } \\
\text { tratamento para a lesão por pressão, }\end{array}$ \\
\hline 7 & $\begin{array}{l}\text { GALVÃO NS, et al. } \\
2017\end{array}$ & $\begin{array}{l}\text { Conhecimentos da equipe de } \\
\text { enfermagem sobre prevenção } \\
\text { de úlceras por pressão. }\end{array}$ & $\begin{array}{l}\text { Os resultados observaram que as médias } \\
\text { globais de acertos foram } 63,4 \% \text { para os } \\
\text { técnicos/auxiliares e } 51,4 \% \text { para os } \\
\text { enfermeiros. }\end{array}$ & $\begin{array}{l}\text { Conclui-se que é importante a } \\
\text { capacitação entre a equipe de enfermagem, } \\
\text { devido ao déficit de conhecimento sobre a } \\
\text { prevenção das lesões por pressão. }\end{array}$ \\
\hline 8 & $\begin{array}{l}\text { SANTANA WS, et al. } \\
2014\end{array}$ & $\begin{array}{l}\text { Prevalência de Úlcera por } \\
\text { Pressão em Idosos com } \\
\text { Imobilidade Prolongada em } \\
\text { Domicílio. }\end{array}$ & $\begin{array}{l}\text { Os resultados evidenciaram que a faixa } \\
\text { etária predominante foi de } 71 \text { e } 81 \text { anos } \\
(42,9 \%) \text { da amostra pesquisada. }\end{array}$ & $\begin{array}{l}\text { Conclui-se que a ocorrência de LPP em } \\
\text { idosos hospitalizados é bem maior do que } \\
\text { em outras faixas etárias, evidenciando a } \\
\text { Necessidade de intensificação da educação } \\
\text { em saúde e ações de acompanhamento dos } \\
\text { idosos em situações de risco para } \\
\text { desenvolvimento de LPP, visando } \\
\text { prevenção e melhora da qualidade de vida. }\end{array}$ \\
\hline 9 & $\begin{array}{l}\text { ROGENSK NMB e } \\
\text { KURCGANT P, } 2012\end{array}$ & $\begin{array}{l}\text { Incidência de Úlceras por } \\
\text { pressão após a implementação } \\
\text { de um protocolo de prevenção. }\end{array}$ & $\begin{array}{l}\text { Os resultados demonstraram que as } \\
\text { instituições que adotam o protocolo de } \\
\text { prevenção de lesão por pressão obtêm uma } \\
\text { melhor qualidade de vida para os pacientes. }\end{array}$ & $\begin{array}{l}\text { Conclui-se que os protocolos de Prevenção } \\
\text { são ferramentas fundamentais er } \\
\text { impacto no controle da incidência de lesão } \\
\text { por pressão, quando } \\
\text { sistematicamente. }\end{array}$ \\
\hline 10 & SANTOS CT, et al, 2013 & $\begin{array}{l}\text { Indicador de qualidade } \\
\text { assistencial de úlcera por } \\
\text { pressão: análise de prontuário e } \\
\text { de notificação de incidente. }\end{array}$ & $\begin{array}{l}\text { Os resultados evidenciaram que há uma } \\
\text { evolução positiva em relação à assistência da } \\
\text { enfermagem em pacientes com lesão por } \\
\text { pressão, entretanto os achados observaram } \\
\text { uma subnotificação dos dados. }\end{array}$ & $\begin{array}{lllll}\text { Conclui-se } & \text { que } & 0 & \text { diagnóstico } & \text { de } \\
\text { enfermagem } & \text { mais frequente foi risco } & \text { de } \\
\text { infecção. } & & & & \end{array}$ \\
\hline 11 & LIMA AFC, et al., 2016 & $\begin{array}{l}\text { Tecnologia no cuidado ao } \\
\text { paciente internado numa } \\
\text { unidade de clínica médica: } \\
\text { segurança na prevenção de } \\
\text { lesão por pressão. }\end{array}$ & $\begin{array}{l}\text { Os resultados mostraram que o uso da } \\
\text { tecnologia na prática dos profissionais de } \\
\text { saúde vem sendo empregado como uma } \\
\text { ferramenta auxiliadora no processo de } \\
\text { cuidar, visto que dinamiza a assistência, } \\
\text { tomando, por vezes, o cuidar mais eficaz. }\end{array}$ & $\begin{array}{l}\text { Conclui-se que a capacidade humana é } \\
\text { importante em buscar inovações capazes de } \\
\text { modificar seu cotidiano, visando uma melhor } \\
\text { qualidade de vida e uma maior realização } \\
\text { profissional. }\end{array}$ \\
\hline
\end{tabular}

Fonte: Pesquisa direta, 2017.

REAS/EJCH | Vol.Sup.30 | e1440 | DOI: https://doi.org/10.25248/reas.e1440.2019 Página 7 de 9 


\section{DISCUSSÃO}

\section{Incidência e prevalência de lesão por pressão em pacientes hospitalizados}

Essa categoria foi formada a partir dos trechos selecionados de seis artigos que compõe a amostra da pesquisa, os quais evidenciam a incidência e prevalência de lesão por pressão em pacientes hospitalizados. A utilização de protocolos nos tratamentos de pacientes hospitalizados vem se tornando eficaz e alcançado vários segmentos. Os autores analisados são unânimes em relação a eficácia e aos benefícios que a padronização do atendimento a esses pacientes proporciona.

Os achados de Matos LS, et al. (2010), evidenciaram que metade das lesões apareceram a partir do $2^{\circ}$ dia de avaliação. Segundo a Escala de Braden, mais da metade dos pacientes apresentavam alto risco de desenvolver LPP. Os autores puderam concluir que as incidências podem ser reduzidas se houver estratégias eficaz, tais como a adoção de protocolos de atendimentos.

Já os achados de Olkosk E e Assis GM (2016), verificaram que aplicação de medidas simples como o ajuste da cabeça e dos tornozelos dos pacientes contribuem para a redução de casos de LPP. Os autores concluíram que a efetividade deste tipo de abordagem é dependente da participação ativa da equipe na discussão de medidas a serem aplicadas e de recursos disponíveis.

Para os autores Galvão NS, et al. (2017), observaram que os conhecimentos da equipe de enfermagem sobre prevenção das lesões por pressão são primordiais para o atendimento e a diminuição das incidências, uma vez que com uma equipe qualificada e com o aparato tecnológico ao seu dispor, o paciente ganha mais qualidade de vida. Assim, os autores concluíram que é importante a capacitação entre a equipe de enfermagem, devido ao déficit de conhecimento sobre a prevenção das lesões por pressão.

Contudo, ao examinar os estudos de Santana WS, et al. (2014) foi possível observar que os idosos são mais acometidos dessa comorbidade devido a sua fragilidade e vulnerabilidade física e emocional, haja vista ser esse o período em que as pessoas precisam de mais ajuda médica, evidenciando a necessidade de intensificação da educação em saúde e ações de acompanhamento dos idosos em situações de risco para desenvolvimento de LPP, visando prevenção e melhora da qualidade de vida.

Os estudos de Rogensk NMB e Kurcgant $P$ (2012) demonstraram que as equipes de enfermagem que adotam o protocolo de prevenção de lesão por pressão obtêm uma melhor qualidade de vida para os pacientes. Concluindo, assim, que os protocolos de prevenção são ferramentas fundamentais e de impacto no controle da incidência de lesão por pressão.

\section{A assistência e cuidado da enfermagem na prevenção de lesão por pressão.}

Essa categoria é formada por seis amostras que evidenciaram a assistência e cuidado da enfermagem na prevenção de lesão por pressão. Ao examinar os achados de Lamão LCL, et al. (2016), observou-se que as lesões mais comuns apareceram entre o $2^{\circ}$ e $04^{\circ}$ dia de avaliação. Segundo a Escala de Braden, mais da metade dos pacientes apresentavam alto risco de desenvolver lesão por pressão. Os autores concluíram que a assistência prestada aos pacientes é desenvolvida através de estratégias de redução destes agravos.

Os estudos de França JRG, et al. (2016), observaram que o gênero masculino apresenta incidência maiores de lesões por pressão, embora não existam dados na literatura que justifiquem essa estatística.

Ao examinar os estudos de Saatkamp F (2012) foi observado que a enfermagem destaca como fator principal para a incidência de lesão por pressão a qualidade dos alimentos ingeridos pelos pacientes, assim como a falta de conhecimento dos familiares sobre as LPP. E o autor conclui que o papel da enfermagem no cuidado e assistência para com o paciente, que deve ser buscado por toda instituição hospitalar.

Para Souza VPS, et al. (2016) a equipe de enfermagem desenvolve ações determinantes na prevenção, promoção e tratamento das LPP, sendo necessária a atuação direta do enfermeiro para a efetividade do tratamento preventivo, moldando os cuidados conforme os fatores integrais e individuais. Os autores concluem que é muito importante que a equipe multidisciplinar reforce as intervenções, interagindo no planejamento e na elaboração de programa de prevenção e tratamento para a lesão por pressão. 
Já os estudos de Santos CT, et al. (2013) observaram que há uma evolução positiva em relação à assistência da enfermagem em pacientes com lesão por pressão, entretanto os achados observaram uma subnotificação dos dados, ou seja, há uma omissão de casos de LPP. Sendo os casos mais notificados são os de infecção. Finalizando, os achados de Lima AFC, et al. (2016) demonstraram que a utilização da tecnologia na prática dos profissionais de saúde vem sendo empregado como uma ferramenta auxiliadora no processo de cuidado, otimizando a assistência, tornando o cuidar eficaz.

\section{CONCLUSÃO}

A pesquisa demonstrou, através da revisão integrativa da literatura, que a assistência e o cuidado da enfermagem fazem toda a diferença no tratamento e recuperação desses pacientes com lesão por pressão, Assim, se faz necessário que estas tenham os devidos conhecimentos técnicos científicos para que ofereça as principais medidas de prevenção, como por exemplo, as medidas simples, porém eficazes como: mudanças de decúbito, hidratação da pele, proteção das saliências ósseas e manutenção da higiene do paciente. Contudo, a produção científica evidenciou que há lacunas existentes no conhecimento produzido sobre a temática abordada. E que se faz necessário futuros estudos nesse tema, a fim de aprofundar 0 conhecimento científico.

\section{REFERÊNCIAS}

1. ABNT - Associação Brasileira de Normas Técnicas. NBR 14724: Informação e documentação. Trabalhos Acadêmicos - Apresentação. Rio de Janeiro: ABNT,2002.

2. DOMANSKY RC, BORGES EL. Manual para prevenções de lesão de pele. 2. ed. Rio de Janeiro: Rubio, 2014.

3. FERNANDES LM, CALIRI MHL. Using the Braden and Glasgow scales to predict pressure ulcer risk in patients hospitalized at intensive care units. Rev. Latino-Am. Enfermagem [Internet]. v.16, n 6, nov. Dez; p.78-84, 2016.

4. FRANÇA JRG., et al. Cuidados de Enfermagem na Prevenção de Lesões por Pressão em Unidades de Terapia Intensiva: uma Revisão Sistemática. Revista Brasileira de Saúde Funcional, Cachoeira- Ba, v. 1, n. 11, p.16-31, jun. 2016.

5. GALVÃO NS et al. Conhecimentos da equipe de enfermagem sobre prevenção de úlceras por pressão. Rev Bras Enferm [Internet], v.70, n. 3, mar-abr., p.312- 318, 2017.

6. LAMÃO LCL et al. Cuidados de enfermagem na prevenção de lesão por pressão. Revista Científica Interdisciplinar., Paranaguá, v. 1, n. 9, p.122-181, dez. 2016.

7. LIMA, AFC., et al. Custo direto dos curativos de úlceras por pressão em pacientes hospitalizados. Revista Brasileira de Enfermagem, [s.I.], v. 69, n. 2, p.290-297, abr. 2016. FapUNIFESP (SciELO). http://dx.doi.org/10.1590/0034-7167.2016690212i.

8. MATOS LS, et al. Incidência e prevalência de úlcera por pressão no CTI de um Hospital Público do DF. Rev. Eletr. Enf. [Internet]. 2010 out/dez;12(4):719-26.

9. MARCONI MA, LAKATOS, EM. Técnicas de pesquisa: planejamento e execução de pesquisas, amostragens e técnicas de pesquisa, elaboração, análise e interpretação de dados 7ed. São Paulo: Atlas, 2010.

10. MENDES, KDS et al. Revisão integrativa: método de pesquisa para a incorporação de evidências na saúde e na enfermagem. Texto contexto - enferm. v.17, n.4, pp.758-764, 2008.

11. MINAYO, MCS. O desafio do conhecimento: pesquisa qualitativa em saúde. 13. ed., São Paulo: Hucitec, 2013.

12. OLOSK, E, ASSIS, GM. Aplicação de medidas de prevenção para úlceras por pressão pela equipe de enfermagem antes e após uma campanha educativa. Esc Anna Nery, v. 20, n. 2, p. 363-369, mar/abr.,2016.

13. SAATKAMP, F. Cuidados de enfermagem na prevenção á úlceras por pressão em pacientes hospitalizados. Rev Bras Enferm [Internet], v.15, n. 3, mar- abr., p.312- 318, 2012.

14. SANTANA, WS et al. Prevalência de Úlcera por Pressão em Idosos com Imobilidade Prolongada em Domicílio. Rev Bras Enferm. v. 12, n. 4, p.56-62, 2014.

15. SANTOS, CT et al. Indicador de qualidade assistencial úlcera por pressão: análise de prontuário e de notificação de incidente. Revista gaúcha de enfermagem. Porto Alegre, v. 34, n. 1, p. 111-118, 2013.

16. SILVA RCL. Feridas fundamentos e atualizações em enfermagem. 3o ed. São Paulo: YENDIS, p 57-61. 2011.

17. SOUZA, VPS et al. Assistência de enfermagem na prevenção de lesão por pressão. Rev Bras Enferm [Internet], v.15, n. 3, mar-abr., p.312- 318, 2016.

18. ROGENSK NMB, KURCGANT, P. Incidência de úlceras por pressão após a implementação de um protocolo de prevenção. Rev. Latino-am. Enfermagem, São Paulo, v. 2, n. 20, p.1-7, abr. 2012. 